\title{
Comment on "Linking population-level models with growing networks: A class of epidemic models"
}

\author{
Martin C. J. Bootsma and Odo Diekmann \\ Department of Mathematics, Utrecht University, Budapestlaan 6, P.O. Box 80.0103508 TA Utrecht, The Netherlands
}

(Received 24 November 2005; published 25 July 2006)

\begin{abstract}
In this Comment, we show that the expression for $R_{0}$ as proposed by R. Breban, R. Vardavas, and S. Blower, Phys. Rev. E 72, 046110 (2005) is incorrect and we reveal the underlying conceptual mistake.

DOI: 10.1103/PhysRevE.74.018101

PACS number(s): 89.75.Hc, 87.19.Xx, 87.23.Ge, 05.10.-a
\end{abstract}

Using a Kinetic Monte Carlo (KMC) growing network, Breban et al. [1] calculate an $R_{0}$ value of 1 for a simple birth-death model with growth rate $\beta$ and death rate $\mu$, with $\beta>\mu$. Their calculation is based on the formula

$$
R_{0}=\sum_{Q=0}^{\infty} Q p_{Q}(\infty)
$$

with $Q$ the number of offspring and $p_{Q}(\infty)$ the fraction of individuals with $Q$ offspring when time approaches infinity. However, $R_{0}$ is an average based on sampling at birth, which is not the same as sampling of the extant population. This can easily be shown as follows. A newborn individual lives for an expected amount of time $1 / \mu$ while producing offspring at rate $\beta$, so has an expected number of offspring $\beta / \mu$. The expected number of offspring at age $x$, given that the individual is still alive, equals $\beta x$. The stable age distribution has density,

$$
\beta e^{-\beta x}
$$

(the product of the survival probability $e^{-\mu x}$ and a factor $\beta e^{-(\beta-\mu) x}$ which incorporates the effect that the growth of the population has on the age distribution and guarantees that the integral equals 1). Therefore, the expected number of offspring per individual alive equals

$$
\int_{0}^{\infty} \beta^{2} x e^{-\beta x}=\int_{0}^{\infty} d x \beta e^{-\beta x}=1 .
$$

In fact this result is a reformulation of the Euler-Lotka equation which serves to characterize the population growth rate.
The reduction of $\beta / \mu$ to 1 is due to the overrepresentation of young individuals in a growing population.

The stable age distribution can be derived from the partial differential equation for the density $u(t, x)$ of individuals of age $x$ at time $t$,

$$
u_{t}+u_{x}=-\mu u
$$

as indeed Breban et al. do correctly in the Appendixes B and C. So our comment concerns a conceptual matter, not the technical elaboration. In the epidemic context, $R_{0}$ is, by definition, an average over (a sample of) individuals that are just infected and then followed during their infectious period to count the number of transmissions they bring about. Alternatively one may, in principle, take a snapshot in time and count per infectious individual how many secondary cases they have caused and then calculate the average. In a rising epidemic the latter should equal 1 if the transient effects of the precise way in which the outbreak was started up have died out, but the reduction of the pool of susceptibles by acquired immunity is not yet an issue.

Whenever a network does not have much structure, it can be described by a branching process. The theory for these processes is in very good shape, see, e.g., Ref. [2]. In fact one can use this theory to investigate the likelihood of a minor outbreak despite $R_{0}$ being bigger than 1 (an issue that is not addressed at all in Breban et al. [1]) and even, by considering the backward process, the final size distribution, see Sec. 10.5.2 of Ref. [3].
[1] R. Breban, R. Vardavas, and S. Blower, Phys. Rev. E 72, 046110 (2005).

[2] P. Haccou, P. Jagers, and V. A. Vatutin, Branching Processes: Variation, Growth and Extinction of Populations (Cambridge
University Press, Cambridge, 2005).

[3] O. Diekmann and J. A. P. Heesterbeek, Mathematical Epidemiology of Infectious Diseases: Model Building, Analysis and Interpretation (Wiley, Chichester, 2000). 